\title{
PROSPECTIVA DE LA AGROINDUSTRIA DE PRODUCCIÓN DE FLOR DE CORTE DEL DEPARTAMENTO DEL QUINDÍO BAJO LA METODOLOGÍA DE GODET
}

\section{AGROINDUSTRIAL PRODUCTION OF CUT FLOWERS PROSPECTIVE AT QUINDÍO REGION UNDER THE METHODOLOGY OF GODET}

\author{
Ximena Cifuentes Wchima* \\ Luis Miguel Mejía Giraldo**
}

Cifuentes W, Ximena, Mejía G. Luis M. Sophia Nº - 2011. ISSN: 194-8932 Págs. 184-191.

Recepción: Junio 15 de 2011

Aceptación: Agosto 2 de 2011

\section{RESUMEN}

Se llevó a cabo una investigación prospectiva de la Agroindustria de producción de flores de corte del Departamento del Quindío, la cual comprende la zona marginal alta cafetera y se observó que el entorno de mayor influencia es el tecnológico e influyendo principalmente al entorno económico. Además al realizar el análisis de impacto con el uso del criterio prospectivo se detectó que las variables críticas son la posibilidad de exportación, los altos márgenes de rentabilidad que podrían ser alcanzados y la ubicación de zonas agroecológicas para la producción de flores y follajes, y como variables externas influyentes el estimulo al desarrollo de empresas y el apoyo con políticas de explotación por el Gobierno.

\section{PALABRAS CLAVE}

Agroindustria, Flores de Corte, Análisis Prospectivo, Quindío.

\section{ABSTRAC}

A prospective research of agro industrial production of cut flowers was carried out at Quindío Region, involving the high marginal coffee zone. The environment with a major influence as observed was the technological one which also affects the economic factor. Besides; doing the analysis of impact using the prospective criteria, it was detected the possibility of exportation as a critical variable, as well as the high margins of profitability that could be reached, the location of agro ecological zones for the production of flowers and foliage, and as external impacting variables, the stimulus for business development and the support from government with exploitation policies.

\section{KEY WORDS}

Agroindustry, Cut Flowers, Prospective Analysis, Quindío.

* Decana Facultad de Ingeniería Universidad La Gran Colombia Seccional Armenia. Grupo GIDA, Universidad La Gran Colombia Armenia, Colombia.defingenieria@ugca.edu.co - Colombia.

** Docente investigador Programa Ingeniería Agroindustrial, Grupo GIDA, Universidad La Gran Colombia Armenia, Colombia. miguelmejia99@yahoo.com - Avenida Bolívar No. 7-46 Armenia, Quindío, Colombia. 


\section{INTRODUCCIÓN}

El sector floricultor colombiano ha sido una agroindustria que posee el prestigio de ser un sector exportador que ocupa primeros lugares en la generación de divisas al país dentro del grupo de productos de exportación no tradicional, representando en el año de 1997 el 4.7\% del total de las exportaciones nacionales y el $0.4 \%$ del Producto Interno Bruto (PIB), su crecimiento es de tendencia relativamente constante conservando una tasa promedio de $1.1 \%$ anual (dentro del PIB).

Dado el potencial económico expansivo de dicha agroindustria, se hace necesario el diseño de sistemas de producción agrícolas rentables, con enfoque empresarial como alternativas para el estímulo al desarrollo del agro y creación de empleo rural, a su vez enfocados hacia la protección del medio ambiente a través de la aplicación de principios de sostenibilidad, y que además permita un manejo equitativo de los recursos económicos, siendo el sector floricultor un medio atractivo para lograr tales objetivos, ya que las flores así como el café han sido sectores competitivos dados sus altos niveles de penetración en los mercados mundiales (Mejía, 2003).

A su vez, la misma dinámica global exige un alto compromiso empresarial en función de la competitividad al interior de clusters claramente definidos como en el caso del Quindío en los municipios de Salento y Filandia, principalmente.

Además, se requiere del enfoque o visión de cadenas productivas o clusters, los cuales son definidos como "Un grupo geográficamente próximo de compañías interconectadas e instituciones asociadas, en un campo particular, vinculadas por características comunes y complementarias" (Porter, 1991). Esto incluye proveedores de insumos, clientes, componentes tecnológicos requeridos en el agroecosistema y servicios especializados de asesoría y consultoría, además de la participación de instituciones financieras, canales de distribución bien establecidos, empresas de productos complementarios, instituciones públicas y privadas de educación, información e investigación, agencias supervisoras y reguladoras y asociaciones gremiales (Mejía et al, 2003).
En la actualidad se han generado una serie de herramientas y métodos de predicción y análisis de información que propenden por realizar proyecciones con base en herramientas que brinden un sentido lógico y razonable para quien las aplica. Es de resaltar que para la realización de tales proyecciones se deben definir una serie de escenarios sobre los cuales opera el sistema, como afirma Mora (2007) que son entornos que se valoran a futuro y que se dan dentro de unas condiciones referenciadas por hechos y opiniones de expertos, para que esta metodología califique verdaderamente como de método científico, debe desarrollarse con una metodología científica y no basada en simples conjeturas humanas conjugadas de tal manera; no obstante, así como resalta Godet (1999), se debe manejar solo como una herramienta simple de nivel subjetivo, que es muy útil para iniciar procesos serios de planeación estratégica tecnológica con base en el estado futuro, el presente estudio basado en los planteamientos anteriores se realizó para entender el ecosistema empresarial sobre el cual se desempeña el sector floricultor de flores de corte del Departamento del Quindío, dado que es necesario describir inicialmente los aspectos más relevantes e influyentes provenientes de los entornos sobre los cuales la empresa se desenvolverá, ya que el desconocimiento del macroambiente puede arriesgar la integridad de la agroindustria bajo estudio.

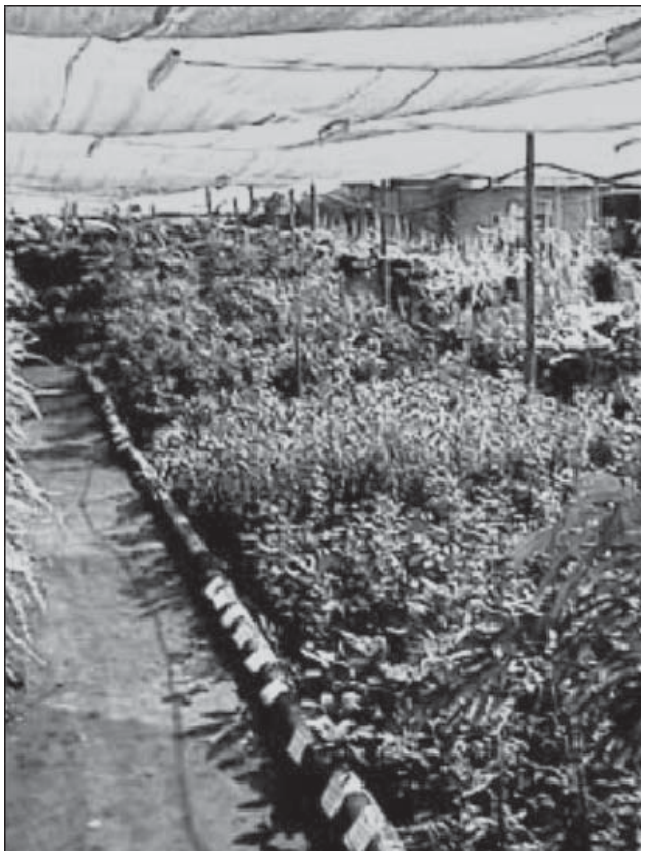

Fuente. Mejía, 2000 
Todo lo anterior se lleva a cabo por medio del análisis estructural que es una herramienta de estructuración de una reflexión colectiva. Ofrece la posibilidad de describir un sistema con ayuda de una matriz que relaciona todos sus elementos constitutivos.

Partiendo de esta descripción, este método tiene por objetivo, hacer aparecer las principales variables influyente y dependientes y por ello las variables esenciales a la evolución del sistema, dicha metodología de análisis estructural prospectivo de impactos cruzados es conocida como MIC MAC, la cual adquiere su divulgación entre 1972 y 1974 y se traduce como - Matrice d'Impacts Croisés - Multiplication Appliqueé a un Classement - Matriz de Impacto Cruzado con Multiplicación Aplicada a una Clasificación de variables en un sistema cerrado (Godet,1999) (Duperrin y Godet,1973).

Con base en el uso de la Metodología MIC MAC y en aras de entender la dinámica de entornos que circundan a la floricultura quindiana surge la presente pregunta de investigación: ¿Cuál es la influencia de los diferentes entornos que afectan a la agroindustria de producción de flores en el Departamento del Quindío?, cuestionamiento fundamental para la definición de planes a seguir para el desarrollo, productividad y en última instancia la competitividad de dicho renglón socioeconómico.

\section{METODOLOGÍA}

La Metodología se fundamenta en el establecimiento de la matriz de impacto cruzado con multiplicación aplicada a una clasificación de variables en un sistema cerrado, conocido por sus siglas como MIC MAC.

En primera instancia se determinaron aquellos aspectos asociados a los diferentes entornos:

\section{Entorno económico}

El entorno económico, esta compuesto fundamentalmente de los siguientes aspectos que influyen en la agroindustria:

- Altos Márgenes de Rentabilidad.

- Inversión Inicial Alta.

- Mercado Maduro de Flores.

- Posibilidad de Exportación.

\section{Entorno demográfico y geográfico}

Con respecto al entorno demográfico y geográfico se definieron los siguientes aspectos de mayor relevancia para la agroindustria:

- Zonas Agroecológicas Óptimas: El Departamento del Quindío posee zonas marginales (de difícil adaptabilidad para las plantas) para la explotación del cultivo del café y plátano, pero favorables para la producción de flores y follajes de corte, ubicadas en los municipios de Salento y Filandia.

- Buen Estado de Vías.

- Ubicación Estratégica del Quindío.

\section{Entorno gubernamental, político y jurídico}

Con respecto al entorno en mención, la empresa esta influenciada por los siguientes aspectos, principalmente:

- Posibilidad de crear Alianzas para Investigar con Universidades de la Región.

- Apoyo con Políticas de Explotación.

- Incentivo a la Capitalización Rural.

- Estimulo al Desarrollo Empresarial.

\section{Entorno sociocultural}

Los aspectos socioculturales que pueden influir en mayor grado en el establecimiento de la empresa agrícola en la zona marginal alta cafetera en el departamento son:

- Generación de Empleo.

- Posibilidad de Equidad de Género.

- Falta de Cultura Floricultora.

- Nivel de Escolaridad Medio-Bajo.

\section{Entorno tecnológico}

Al analizar el entorno tecnológico que influye directamente sobre la agroindustria, se determinaron 5 aspectos que influyen directamente sobre el desarrollo de la empresa productor de flores y follajes, estos son:

- Fundamentación en Investigación y Desarrollo $(I+D)$.

- Información de Vanguardia.

- Diseño de Sistemas de Información.

- Desarrollo de Tecnologías de Cultivos bajo Cubierta.

- Desarrollo en Biotecnología In Vitro.

\section{Entorno ecológico}

- Riesgo de Contaminación Ambiental.

- Biodiversidad.

- Corredor Biológico en la Región. 


\section{Identificación de los Entornos influyentes en la Empresa}

Para identificar los entornos con mayor influencia sobre la empresa se construyó una matriz de impacto y un diagrama de influencia, con base en la metodología citada por el American Council para la Universidad de las Naciones Unidas (2002) de los diferentes entornos que podrían afectar directa o indirectamente la agroindustria. No obstante, dichos matriz y diagrama solo determinan la influencia de cada uno de estos entornos analizados en la agroindustria pero de manera general. Se empleo con base en dicha metodología una escala de 0 a 3 con modificación, utilizándose el signo mas para un efecto incremental o positivo (a medida que aumenta " $x$ ", aumenta " $y$ ") y el menos cuando el efecto es detrimental sobre el entorno afectado (a medida que aumenta " $x$ ", disminuye " $y$ ").

Escala de Valoración de la matriz para diagrama de influencia:

\begin{tabular}{ll}
\hline Influencia & Valor \\
\hline Alto grado positivo & +3 \\
Mediano grado positivo & +2 \\
Bajo grado positivo & +1 \\
No influencia & 0 \\
Bajo grado negativo & -1 \\
Mediano grado negativo & -2 \\
Alto grado negativo & -3 \\
\hline
\end{tabular}

Posteriormente se realizó una definición de problemas de la agroindustria de flores de corte del Departamento.

Por otro lado se realizó la construcción de la matriz de impacto cruzado para las mismas variables en escalas de 0 (Impacto nulo) a 5 (Alto impacto), con base en la metodología de Godet (1985), para posteriormente establecer la priorización de problemas en críticos (alto impacto y alta dependencia entre ellos), activos (alto impacto pero baja dependencia entre ellos), reactivos (variables dependientes o respuesta a los problemas anteriores) e inertes (problemas de bajo impacto y baja dependencia), lo cual también se conoce como matriz de impacto.

\section{RESULTADOS Y DISCUSIÓN}

Después de realizar el cruce de entornos al interior de la matriz o tabla cruzada y se suman las filas con base en valores absolutos para determinar los entornos con mayor influencia sobre los otros, observándose para la Agroindustria bajo estudio que el entorno de mayor influencia es el Tecnológico, con base en el análisis de las variables por entorno que mayor peso poseen sobre la empresa (Tabla 1, Gráfica 1).

Tabla 1: Cruce de entornos

\begin{tabular}{l|c|c|c|c|c|c|c}
\hline Entorno & Económico & $\begin{array}{l}\text { Demográfico/ } \\
\text { Geográfico }\end{array}$ & $\begin{array}{l}\text { Gubernamental } \\
\text { Político-Jurídico }\end{array}$ & Sociocultural & Tecnológico & Ecológico & Total \\
\hline Económico & $\mathrm{X}$ & 0 & +3 & 0 & 0 & +1 & 4 \\
\hline $\begin{array}{l}\text { Demográfico/ } \\
\text { Geográfico }\end{array}$ & +1 & $\mathrm{X}$ & +1 & 0 & 0 & +1 & 3 \\
\hline $\begin{array}{l}\text { Gubernamental- } \\
\text { Político-Jurídico }\end{array}$ & +2 & 0 & $\mathrm{X}$ & +3 & +1 & 0 & 6 \\
\hline Sociocultural & 0 & 0 & +1 & $\mathrm{X}$ & 0 & 0 & 1 \\
\hline Tecnológico & +3 & +2 & +2 & +1 & $\mathrm{X}$ & +1 & 9 \\
\hline Ecológico & 0 & 0 & 0 & 0 & 0 & $\mathrm{X}$ & 0 \\
\hline
\end{tabular}

Fuente. Los Autores, 2009 
El diagrama de influencia es:

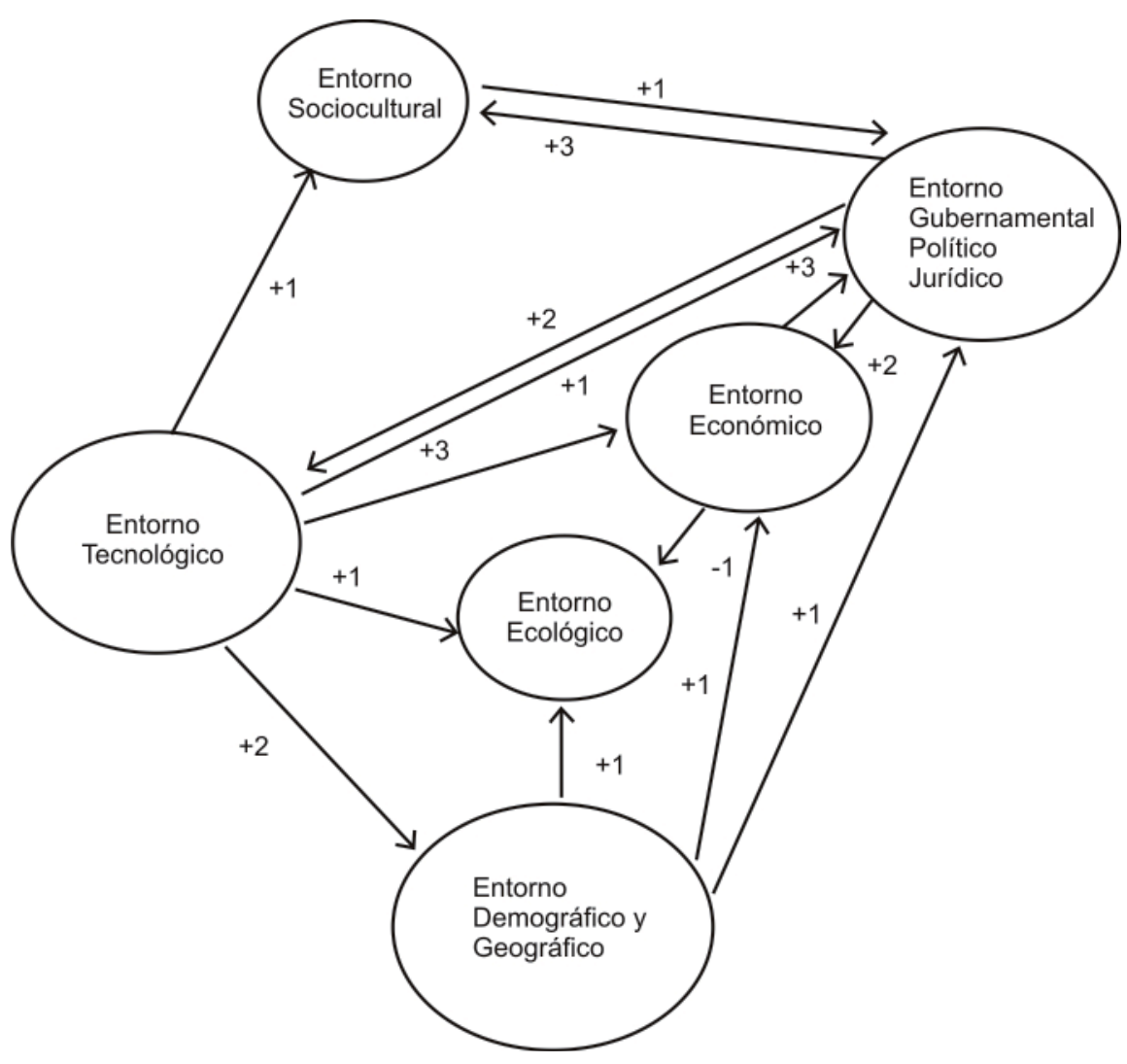

Gráfica 1: Diagrama de Influencia, Fuente Los Autores, 2009

Como se aprecia en el diagrama, el entorno con mayor influencia es el tecnológico, dado que afecta positivamente a todos los demás entornos, siendo el mas influenciado el entorno económico; es decir, que trabajando sobre los 5 aspectos tecnológicos mencionados con antelación puede influir positivamente sobre los márgenes de rentabilidad, la inversión inicial, el conocimiento de los mercados y posibilita la exportación de las flores y follajes.

Le siguen el entorno demográfico y geográfico que influye positivamente pero en bajo grado sobre los entornos económico, ecológico y gubernamental, político y jurídico; el entorno gubernamental, político y jurídico influye positivamente y en alto grado sobre el entorno sociocultural y en mediano grado sobre los entornos tecnológico y económico.
En contraste a lo anterior, el entorno ecológico es afectado por los entornos tecnológico y demográfico y geográfico de manera positiva en bajo grado y de manera negativa en bajo grado por el entorno económico.

Se puede afirmar entonces, que la agroindustria debe estar fundamentada en los componentes del entorno tecnológico para posibilitar su crecimiento empresarial, ya que sobre los cuales puede tener control.

Es de agregar que se debe prestar atención constante a los entornos demográfico y geográfico y gubernamental, político y jurídico porque influyen positivamente sobre el desarrollo empresarial de la agroindustria; mientras que el entorno ecológico requiere de sistema de monitoreo y control dado que a pesar de no influir sobre los demás entornos 
en el macroambiente, garantiza la sostenibilidad ambiental que es complemento fundamental de la equidad y competitividad en el mediano y largo plazo.

Aunque se debe tener en cuenta un comportamiento presentado en el diagrama de posible efecto encadenado de entornos, donde el entorno tecnológico influye en alto grado sobre el entorno económico, este a su vez influye en alto grado sobre el entorno gubernamental, político y jurídico y este a su vez influye sobre el entorno sociocultural, ratificando la importancia del entorno tecnológico para la agroindustria, dado que si este falla puede afectar directamente e indirectamente a los demás.

Posteriormente se realizo la matriz de impacto para determinar el comportamiento de las variables provenientes de los diferentes entornos, las cuales son:

\begin{tabular}{ll}
\hline Entorno/Variables \\
\hline & Entorno Económico \\
A & Altos márgenes de rentabilidad \\
B & Inversión inicial alta \\
C & Mercado de flores maduro \\
D & Posibilidad de Exportación \\
\hline
\end{tabular}

\begin{tabular}{ll}
\hline & Entorno Demográfico y Geográfico \\
E & Zonas agroecológicas óptimas \\
F & Buen estado de vías \\
G & Ubicación estratégica del Quindío
\end{tabular}

\begin{tabular}{cl}
\hline H & $\begin{array}{l}\text { Entorno Gubernamental, Político y Jurídico } \\
\text { Posibilidad de crear alianzas para investigar con } \\
\text { universidad }\end{array}$ \\
I & $\begin{array}{l}\text { Apoyo con políticas de explotación } \\
\text { Incentivo a la capitalización rural }\end{array}$ \\
K & Estímulo al desarrollo empresarial \\
\hline & Entorno Sociocultural \\
L & Generación de Empleo \\
M & Posibilidad de equidad de género \\
$\mathrm{N}$ & Falta de cultura floricultora \\
$\mathrm{O}$ & Nivel de escolaridad medio-bajo \\
\hline & Entorno Tecnológico \\
$\mathrm{P}$ & Fundamentación en l+D \\
$\mathrm{Q}$ & Información de Vanguardia \\
$\mathrm{R}$ & Diseño de Sistemas de Información \\
$\mathrm{S}$ & Desarrollo de Tecnología de cultivos bajo cubierta \\
$\mathrm{T}$ & Desarrollo en biotecnología In Vitro \\
\hline & Entorno Ecológico \\
$\mathrm{U}$ & Riesgo de contaminación ambiental \\
$\mathrm{V}$ & Biodiversidad \\
$\mathrm{W}$ & Corredor biológico en la región \\
\hline
\end{tabular}

\section{La matriz cruzada obtenida fue la siguiente:}

\begin{tabular}{|c|c|c|c|c|c|c|c|c|c|c|c|c|c|c|c|c|c|c|c|c|c|c|}
\hline Variable & a & $b$ o & c & d $E$ & $f$ & g & $\mathrm{h}$ & $i$ & k & I & $m$ & $n$ & 0 & $p$ & $q$ & $r$ & $s$ & t & $\mathrm{u}$ & $\mathrm{v}$ & W & Total Impacto \\
\hline a & $*$ & 0 & 0 & 5 & 1 & 1 & 3 & 5 & 5 & 3 & 1 & 0 & 0 & 0 & 1 & 3 & 5 & 5 & 3 & 1 & 0 & 47 \\
\hline$b$ & 5 & $*$ & 0 & 3 & 0 & 0 & 1 & 5 & 5 & 5 & 3 & 3 & 0 & 0 & 0 & 0 & 0 & 0 & 1 & 0 & 0 & 36 \\
\hline c & 5 & $5{ }^{*}$ & $\star$ & 5 & 3 & 3 & 0 & 3 & 3 & 1 & 0 & 0 & 0 & 1 & 5 & 3 & 3 & 3 & 0 & 0 & 0 & 45 \\
\hline$d$ & 5 & \begin{tabular}{|l|l}
3 & 5 \\
\end{tabular} & 5 & 1 & 3 & 3 & 0 & 3 & 3 & 3 & 3 & 1 & 3 & 0 & 5 & 5 & 0 & 0 & 3 & 1 & 0 & 53 \\
\hline e & 5 & 0 & 0 & 5 & 1 & 5 & 3 & 5 & 5 & 1 & 0 & 0 & 0 & 1 & 0 & 1 & 5 & 5 & 3 & 5 & 5 & 58 \\
\hline$f$ & 3 & 1 & 5 & 3 & ${ }^{*}$ & 3 & 0 & 1 & 0 & 0 & 0 & 0 & 0 & 0 & 0 & 0 & 0 & 0 & 5 & 5 & 0 & 26 \\
\hline 9 & 3 & 1 & 3 & 5 & 55 & 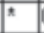 & 0 & 1 & 3 & 0 & 0 & 0 & 0 & 0 & 0 & 0 & 1 & 0 & 1 & 3 & 3 & 35 \\
\hline \multirow[t]{3}{*}{$h$} & 3 & 1 & 1 & 5 & 0 & 0 & $*$ & 3 & 1 & 0 & 0 & 3 & 5 & 5 & 5 & 3 & 3 & 5 & 0 & 0 & 0 & 49 \\
\hline & 3 & 5 & 3 & \begin{tabular}{l|l}
5 & 3 \\
\end{tabular} & \begin{tabular}{|l|}
31 \\
\end{tabular} & 3 & 0 & $\pi$ & 35 & 5 & 5 & 1 & 0 & 0 & 0 & 3 & 0 & 0 & 0 & 0 & 0 & 45 \\
\hline & 5 & 5 & 1 & \begin{tabular}{l|l}
5 & 3 \\
\end{tabular} & $\begin{array}{ll}3 & 1 \\
\end{array}$ & 3 & 0 & 3 & 5 & 5 & 3 & 0 & 0 & 0 & 0 & 0 & 0 & 0 & 0 & 0 & 0 & 39 \\
\hline \multirow[t]{2}{*}{ k } & 5 & \begin{tabular}{|l|l}
31 \\
\end{tabular} & 1 & \begin{tabular}{l|l}
5 & 3 \\
\end{tabular} & $\begin{array}{ll}3 & 1 \\
\end{array}$ & 0 & 0 & 3 & 5 & 5 & 5 & 3 & 3 & 3 & 1 & 3 & 3 & 3 & 1 & 0 & 0 & 56 \\
\hline & 5 & 3 & 0 & 5 & $\begin{array}{ll}0 & 0\end{array}$ & 0 & 0 & 5 & 5 & $\pi$ & 5 & 0 & 0 & 0 & 0 & 0 & 0 & 0 & 0 & 0 & 0 & 33 \\
\hline$n$ & 1 & 0 & 0 & 0 & $\begin{array}{ll}0 & 0 \\
\end{array}$ & 0 & 0 & 5 & 5 & 5 & ${ }^{*}$ & 1 & 3 & 0 & 0 & 0 & 0 & 0 & 0 & 0 & 0 & 30 \\
\hline$\pi$ & 5 & 1 & 5 & 5 & $\begin{array}{ll}0 & 0 \\
\end{array}$ & 0 & 3 & 1 & 3 & 3 & 1 & $\pi$ & 0 & 0 & 0 & 0 & 1 & 1 & 0 & 0 & 0 & 34 \\
\hline 0 & 0 & 0 & 0 & \begin{tabular}{l|l}
3 & 3 \\
\end{tabular} & 0 & 0 & 5 & 3 & 3 & 3 & 3 & 5 & $*$ & 1 & 0 & 0 & 3 & 1 & 5 & 3 & 0 & 46 \\
\hline$p$ & 5 & 3 & 0 & 5 & 0 & 0 & 5 & 3 & 5 & 1 & 0 & 3 & 1 & $*$ & 5 & 5 & 5 & 5 & 3 & 3 & 3 & 70 \\
\hline 9 & 5 & 30 & 0 & \begin{tabular}{l|l}
5 & 3 \\
\end{tabular} & 0 & 3 & 5 & 3 & 1 & 0 & 0 & 1 & 0 & 3 & k & 5 & 1 & 1 & 0 & 0 & 0 & 42 \\
\hline 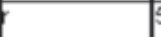 & 5 & 3 & 1 & 3 & 1 & 0 & 0 & 3 & 1 & 0 & 0 & 0 & 3 & 3 & 5 & $*$ & 1 & 1 & 0 & 0 & 0 & 32 \\
\hline \multirow{2}{*}{$s$} & 5 & 51 & 1 & 55 & 1 & 5 & 0 & 3 & 3 & 1 & 0 & 1 & 3 & 1 & 1 & 1 & $*$ & 1 & 3 & 3 & 0 & 49 \\
\hline & 5 & 5 & 1 & 3 & 0 & 5 & 5 & 3 & 3 & 3 & 3 & 0 & 3 & 5 & 3 & 1 & 3 & $\pi$ & 1 & 5 & 3 & 64 \\
\hline$\mu$ & 0 & 1 & 0 & 5 & 0 & 0 & 0 & 3 & 1 & 0 & 0 & 5 & 5 & 3 & 1 & 0 & 5 & 5 & * & 5 & 5 & 49 \\
\hline$v$ & 3 & 1 & 0 & 5 & 0 & 5 & 0 & 1 & 0 & 0 & 0 & 0 & 0 & 3 & 5 & 5 & 0 & 0 & 5 & * & 5 & 41 \\
\hline$w$ & 0 & 0 & 0 & 3 & $\begin{array}{ll}30 \\
0\end{array}$ & 1 & 0 & 0 & 0 & 0 & 0 & 0 & 0 & 1 & 5 & 5 & 3 & 3 & 5 & 5 & * & 34 \\
\hline \multirow{2}{*}{\begin{tabular}{l|} 
Total \\
Dependencia
\end{tabular}} & 81 & 492 & 279 & \begin{tabular}{l|l}
96 & 54
\end{tabular} & 5418 & 40 & 306 & 656 & 636 & \begin{tabular}{l|l}
50 & 44
\end{tabular} & 432 & 27 & 29 & 30 & 42 & & 42 & & 39 & & 24 & 44 \\
\hline & & & & & & & & & & & & & & & & & & & & & & \\
\hline
\end{tabular}




\section{La Gráfica de Matriz de Impacto (Gráfica 2) muestra la ubicación de las variables en los diferentes cuadrantes:}

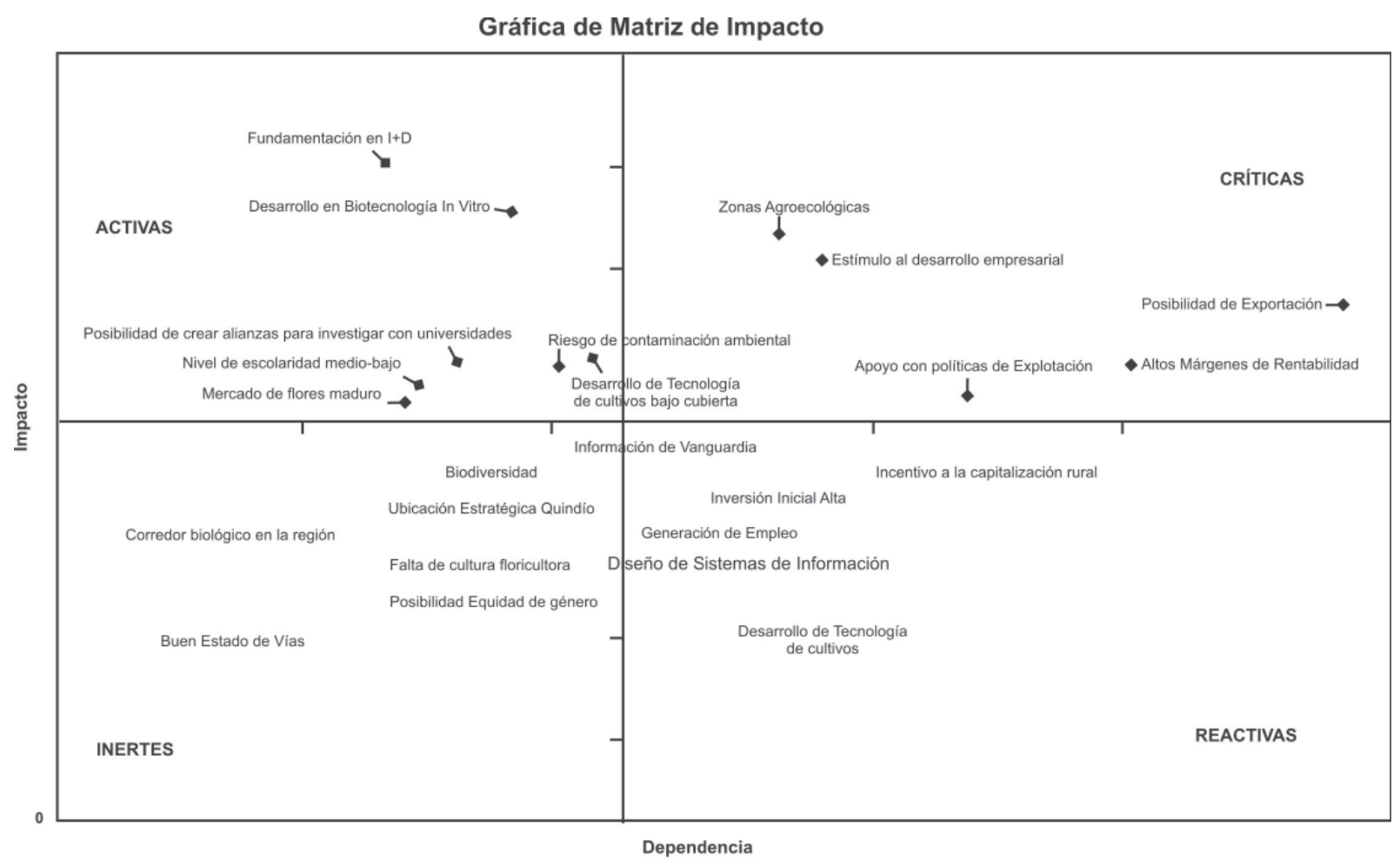

Gráfica 2: Matriz de Impacto Fuente Los Autores, 2009

Los resultados obtenidos en el Gráfico 2 están resumidos en la siguiente tabla:

\begin{tabular}{ll}
\hline Tipo de Variable & Variable \\
\hline Activas & *fundamentación en I+D \\
& *Desarrollo en biotecnología In Vitro \\
& * $P$ osibilidad de crear alianzas \\
& para investigar con universidades \\
& *Nivel de escolaridad medio-bajo \\
& *Mercado de flores maduro \\
& *Riesgo de contaminación ambiental \\
& *Desarrollo de tecnología de cultivos \\
& bajo cubierta \\
\hline Criticas & *Zonas agroecológicas \\
& *Estimulo al desarrollo empresarial \\
& * Posibilidad de exportación \\
& *Altos márgenes de rentabilidad \\
& *Apoyo con políticas de explotación \\
\hline
\end{tabular}

\begin{tabular}{ll}
\hline Tipo de Variable & Variable \\
\hline Reactivas & ${ }^{*}$ Inversión inicial alta \\
& ${ }^{*}$ Incentivo a la capitalización rural \\
& *Generación de empleo \\
\hline Inertes & *Bodiversidad \\
& ${ }^{*}$ Corredor biológico en la región \\
& ${ }^{*}$ Ubicación estratégica del Quindío \\
& *Falta de cultura floricultora \\
& * *osibilidad de equidad de genero \\
& *Información de váas \\
& *Diseño de sistemas de información \\
&
\end{tabular}


Las variables activas que requiere trabajar de manera periódica la agroindustria dada la baja dependencia pero alto impacto son la investigación de los mercados de flores, la creación de alianzas estratégicas con las universidades de la región, en cuanto al entorno tecnológico (quien es el más influyente con base en el diagrama de influencia) se determinó que las variables fundamentación en I+D, Biotecnología In vitro y Desarrollo de Tecnologías bajo Cubierta son aquellas que la agrocadena debe reforzar.

Existen otras variables activas que no deben ser menospreciadas como son nivel de escolaridad medio-bajo y el riesgo de impacto ambiental, como variables que analizadas al nivel de entorno se enmascaraban, pero muestran su influencia en el ámbito individual.

Con respecto a las variables críticas; es decir, aquellas de alta dependencia y alto impacto y que requieren un trabajo similar a las variables activas son la posibilidad de exportación, los altos márgenes de rentabilidad que podrían ser alcanzados, la ubicación de zonas agroecológicas optimas para la producción de flores y follajes, y como variables exógenas influyentes el estimulo al desarrollo empresarial y el apoyo con políticas de explotación por parte del entorno gubernamental.

Es de agregar que al trabajar sobre las variables anteriores (activas y críticas) reaccionan las variables inversión inicial alta (disminución del riesgo de inversión), el incentivo a la capitalización rural por parte del entorno gubernamental y al nivel sociocultural se resalta la generación de empleo.

En cuanto a las variables inertes, aunque pueden influir en la empresa no afectan adversamente el pleno desarrollo de la misma y se pueden suplir a medida que la agroindustria va madurando, a partir del anterior análisis estas son la biodiversidad del Quindío, corredor biológico en la región, ubicación estratégica del departamento, falta de cultura floricultora, posibilidad de equidad de género, buen estado de las vais, información de vanguardia y diseño de sistemas de información.

\section{CONCLUSIONES}

- El entorno tecnológico ejerce fuerte influencia sobre el desarrollo de la agroindustria de flores de corte al interior del Departamento del Quindío.

- Se requiere del establecimiento y consolidación de la agrocadena al interior del Departamento para que los productores aprovechen aspectos como economías de escala, Investigación y Desarrollo y establecer posibilidades de expansión de su mercado hacia aquellos de carácter externo.

- Se requiere del establecimiento de zonas agroecológicas óptimas para la producción de flores de corte en el Departamento.

\section{RECOMENDACIONES}

- Establecer investigación de los mercados de flores actuales y potenciales así como la creación de alianzas estratégicas con las universidades de la región.

- En cuanto al entorno tecnológico se requiere un fortalecimiento en Investigación y Desarrollo $(I+D)$, además de desarrollo de tecnologías bajo Cubierta e investigaciones en el área de la Biotecnología Vegetal In vitro.

\section{REFERENCIAS BIBLIOGRÁFICAS}

DUPERRIN, J.C; GODET, Michel (1973). Méthode de Hierarchisation des Elements d'un Systeme, rapport économique du CEA R-45-41 Comunidad Europea, Francia.

GODET, Michel (1985). Prospective et Planification Estratégique. Editorial Económica, Francia. ISBN: 2717809392.

GODET; Michel (1999). De la anticipación a la Acción. Editorial Alfaomega Marcombo Boixerau Editores, Editorial Alfaguara, Colombia. ISBN: 958-682-004-1

MEJÍA, Luis Miguel (2003). Establecimiento de un Sistema de Comercialización de Flores de Corte para la Zona Marginal Alta Cafetera del Departamento del Quindío, Universidad Santo Tomás.

MORA, Alberto (2007). Pronósticos de Demanda e Inventarios, Métodos Futurísticos. Editorial AMG, Colombia. ISBN 978958-44-0233-2

PORTER, Michael (1991). La Ventaja Competitiva de las Naciones. 